\title{
Roillo: Creating a Social Robot for Playrooms
}

\author{
Marek P. Michalowski \\ Carnegie Mellon University \\ Robotics Institute \\ Pittsburgh, PA 15213 \\ michalowski@cmu. edu
}

\author{
Selma Sabanovic \\ Rensselaer Polytechnic Institute \\ Science \& Technology Studies \\ Troy, NY 12180 \\ sabanserpi.edu
}

\author{
Philipp Michel \\ Carnegie Mellon University \\ Robotics Institute \\ Pittsburgh, PA 15213 \\ pmichelecs.cmu.edu
}

\begin{abstract}
In this paper, we introduce Roillo, a social robotic platform for investigating, in the context of children's playrooms, questions about how to design compelling nonverbal interactive behaviors for social robots. Specifically, we are interested in the importance of rhythm to natural interactions and its role in the expression of affect, attention, and intent. Our design process has consisted of rendering, animation, surveys, mechanical prototyping, and puppeteered interaction with children.
\end{abstract}

\section{INTRODUCTION}

Face-to-face social interaction between humans consists of both nonverbal and verbal behaviors. While we, as interactors and observers, are often more conscious of the latter, the former are critical for enabling a comfortable and wellregulated engagement between two interactors. In the context of building socially interactive robots, much research has focused on how a robot's appearance and physical features might enable the generation of appropriate nonverbal cues [10]. However, less work has been devoted to the temporal qualities of a robot's behavior and how they should relate to the nonverbal behaviors of a human interactor.

In this paper, we introduce Roillo (Fig. 1), a social robotic platform for investigating, in the context of children's playrooms, questions about how to design compelling nonverbal interactive behaviors for social robots. Our backgrounds are in robotics, computer science, social science, and psychology, and our work draws from and aims to contribute to these different disciplines. We first review research involving robots built for interaction with children, as we have been inspired by the applications for which these systems were designed. We then review work in the observation and modeling of interactional rhythm as the basis for our goal of investigating theoretical questions related to the temporal processes underlying natural face-to-face social interaction.

Roillo is intended to serve as a platform for performing experiments in rhythmicity, interactional synchrony, the expression of affect and attention through motion, and deictic gesturing. Up to this point, we have followed a sequential design process involving a variety of exploratory activities: rendering, animation, surveys, physical prototyping, and observation and analysis of interactions with children. We present an account of this process, describe what we have learned, and discuss what we intend to do in the future.

\section{MOtivation \& RELATED WORK}

\section{A. Social robots \& children}

Many researchers in the field of face-to-face human-robot social interaction have developed robots explicitly for use with children. Their motivations are varied, and projects

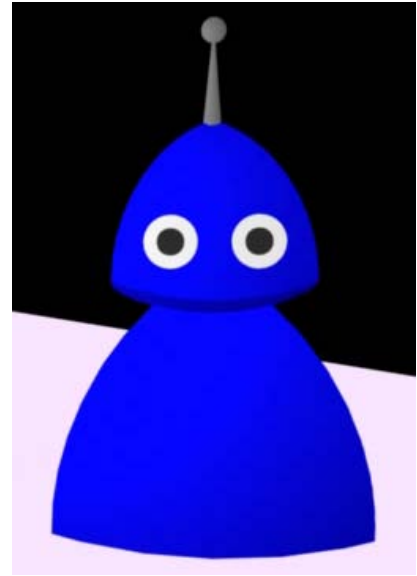

Fig. 1. A rendering of Roillo.

often have multiple stated goals. In some cases, the object of study is development and learning in children; it is investigated either by implementing cognitive developmental models on robotic systems (also known as epigenetic robotics) or by observing (often disabled) children's interactions with social artifacts. Alternatively, the researcher's primary goal might be the development of social robots; this is advanced either through the implementation of a human-like developmental process (e.g. using caregivers as a scaffold) or through iterative testing and evaluation with an audience of children. These goals can be complementary and are frequently combined in the mission statements of social robotics projects.

Infanoid [17] is an upper-torso child-like humanoid that serves as a platform for implementing models of eye contact and joint attention. The Keepon project [18] places a greater emphasis on the observation of children interacting with the robot and asks, for example, what qualities (of robot or child) lead to perception of the robot as a moving object, a living creature, or a social being. Nico [8], another upper-torso baby robot, serves as a test-bed for the implementation of cognitive models that can be evaluated in the same types of experimental settings used in the study of children.

Muu [22] was designed to explore the efficacy of principles of minimal design and ecological psychology for creating socially interactive "artificial creatures." The AuRoRa project [9] also explores the use of robotic toys in therapy for children with autism. They have investigated imitation, turn-taking, narrative comprehension, and group interaction 
in the context of long-term clinical trials. Similarly, the Robotism project [20] explores the use of different mobile robot designs in the rehabilitation of children with autism. Plaisant's storytelling robot [24] is a medium for children in physical as well as psychological rehabilitation to act out emotional stories.

Our work differs from the described approaches in that our primary motivation is not, at this point, learning about human development or developmental disorders. We are interested in the spatiotemporal foundations of social interaction, and children are our audience because we believe that they will be most receptive to the types of tasks and activities that we envision for Roillo.

\section{B. Robots in rhythmic interaction}

Psychological and sociological research suggests that interaction rhythms are elementary and pervasive organizing principles for social interaction [11], [26], [12] and that rhythmic synchrony (the coupling of rhythmic patterns in verbal and nonverbal behavior) is the basis of cognitive processes such as perception, memory and attention [14], [3]. Studies of infant-caretaker interactions show that coordination is critical to the creation of a positive relationship and to the learning of social, cultural and communicative skills [28]. Condon [6] discusses the importance of the matching of movement timing in smooth, friendly communication, and a higher degree of synchronization is generally regarded as a sign of mutual rapport and involvement [4], [19]. Interactional mimicry, feedback and synchrony are also catalysts for emotional contagion [12]. Marked asynchronies within an individual's own behaviors [7], along with abnormal entrainment in interpersonal interactions [5], are often characteristic of pathologies such as autism and schizophrenia.

This attention to the rhythmic characteristics of nonverbal interaction has not been widely adopted in social robotics research. If we neglect to carefully develop internal consistency and interactive synchrony in the nonverbal behavior of robots, their behavior can be experienced as disturbing or offputting by human interactors. General ideas of turn-taking in conversation are widely implemented, but fine-grained rhythmic perception and synchrony by a robot has been difficult to develop. Ogawa [21] used synchronous nonverbal cues in the InterRobot humanoid, which generates facial expressions and bodily movements in response to the speech input of a remote human interactor. The humanoid robot Nico [8] performs drumming synchronized to another person or a conductor as an example of the kind of rhythmic entrainment that might be applied to other social tasks. Synchronized imitation is applied by Andry [1] as a way for robots to learn new types of motion. In existing projects, rhythmic interaction is generally based on auditory cues rather than the perception of embodied movement. However, robots should also be able to "tune in" to the bodily rhythms of their interaction partners as well as to generate such nonverbal behaviors themselves. Penny's Petit Mal [23], which follows simple rules, invokes such a nonverbal rhythmic interaction with people.
A focus on rhythmic interaction, shared forms of organization, coordination, and ritual [11], [7] signifies a shift in social robotics towards creating robots that can perceive, adaptively respond to, and learn to participate in a flow of human socially interactive cues and affordances. Restivo [25] emphasizes rhythmic entrainment, in both speech and body, as the foundation of any socially interactive capabilities that a robot may have. While Trevarthen [27] claims that robots will never be able to think and feel like humans, his insights into the polyrhythmic and melodic nature of human bodily expression can be used to enable robots to perceive, understand, and participate in broader, emergent aspects of human social interaction and culture. Since both self-synchrony (rhythmic matching of movements and vocalizations) and interactional synchrony are important for a normal human-human interaction [16], [7], these capabilities should be considered in designing robots that interact socially with humans.

\section{Deictic gesturing}

Deixis is the process by which language refers to the context in which it is used (i.e. references are made to things in the environment from a certain viewpoint). In faceto-face interaction, spatially directed nonverbal behaviors such as pose, gaze, and concrete deictic (pointing) gestures are important in establishing a mutual understanding of the interactors' shared environment. Much of the literature on deictic gesturing involves the accompaniment of speech by hand, head, and postural movements. In the context of robotics, deictic gesturing is often used to establish a grounding between symbolic labels and perceived objects or to disambiguate language use (see [2] for a recent review).

We are interested in the temporal properties of pointing or reaching as an indication of desire, and the way such gestures may be used by a nonverbal robot. We believe that rhythmicity plays an important role in how such gestures are used and interpreted.

\section{DESIGN: AN ACCOUNT OF OUR PROCESS}

\section{A. Appearance design}

We began with the goal of designing a character with a morphology that would be minimally representative of its interactive capabilities [22]. We do this both to minimize the cost and complexity of the robot and to reduce the inappropriate attributions that might be made to a more visually elaborate form. Johnson et al. [13] have demonstrated that facial features are important cues to intentionality. We believe that a body, a head, and two eyes might represent a minimal set of component elements in a social creature.

A sphere would be the the simplest geometric shape to use for body and head components [18]. However, in the interest of using a shape that would also provide a cue for directionality, we selected the Reuleaux tetrahedron. It is the 3D analog of the Reuleaux triangle, which is the simplest geometric shape of constant diameter after the circle.This shape is used for the body and the head. The eyes, which are to contain wide- and narrow-FOV cameras, consist simply of white discs surrounding black lenses. Roillo also has an 


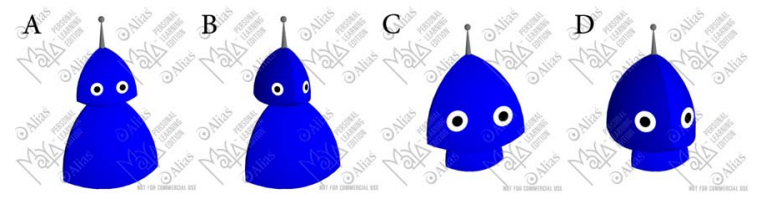

Fig. 2. Initial design candidates, with alternate head orientations and relative head/body sizes.

articulated stick-like "antenna" on top of its head. In contrast to the complexity of multi-joint arms, the antenna provides a simple way of performing deictic gesturing and expressing affect (discussed below).

Given these building blocks, we expected that a particular configuration of the robot would be most appealing to potential interaction parters and would be rated highest in its ability to elicit social interaction. In order to select the relative sizes and orientation of the body components, we conducted an online self-report pretest survey with questions about various appearances that we had rendered using Maya (Fig. 2). Ten participants from the Carnegie Mellon University Robotics Institute were asked to rate the candidate designs in terms of friendliness, "humanlikeness," and apparent age.

The two independent variables that changed between designs were body size and head orientation. We found that users agreed with each other in their evaluations of friendliness, humanlikeness, and age. Head orientation had an effect on the robot's apparent friendliness (the flat face was rated more friendly than the beak-like face by all participants). The survey also suggested that a bigger body makes the robot appear older and more humanlike. These results were in line with our expectations about the candidate designs and led us to select a flat face and a body larger than the head.

\section{B. Behavior design}

Next, we wanted to explore possible behaviors for the expression of affect (emotion) and intent. We animated a number of scripted movements in Maya. In all cases, Roillo sat on a surface next to a red ball. We conducted a second web survey asking about these animations with 48 respondents from the Robotics Institute community.

1) Expressions of affect: To evaluate expressions of affect, we set up an interaction scenario by asking participants to imagine that they had walked up to Roillo and placed a red ball in front of it. They were then shown 6 (randomly ordered) animations of the robot's reaction to the event and asked to rate each in terms of valence and the apparent curiosity of the robot on 5-level Likert scales. We prepared 2 animations each for what we hypothesized were representative expressions of positive, negative, and curious reactions to the ball (e.g. Fig. 3). These animations incorporated head motion (tilting, bobbing, turning), body motion (turning, withdrawing), and antenna motion (wiggling).

Fig. 4 summarizes the valence and curiosity ratings for each of the six affect clips. The expressions we designed as positive and curious are indeed rated significantly higher in terms of valence than those we considered negative

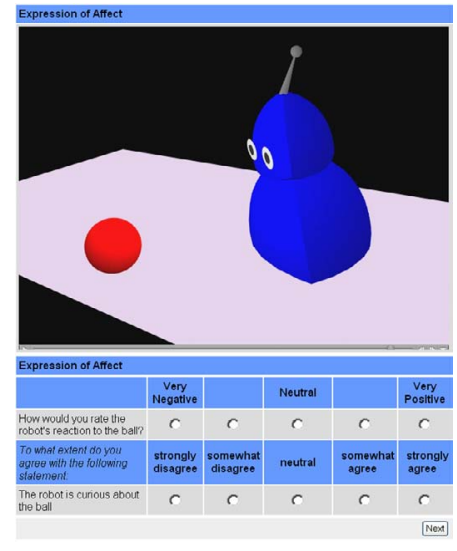

Fig. 3. Roillo exhibiting a "curious" response to the ball.

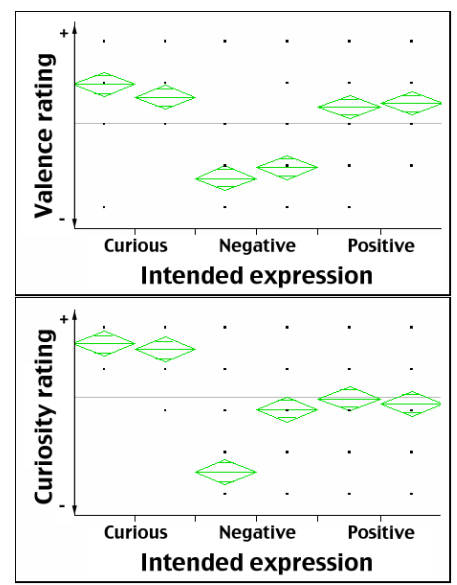

Fig. 4. Means and standard deviations for ratings of valence (top) and curiosity (bottom).

$(\mathrm{F}[5,228]=43.08, \mathrm{p}<.01)$. The behaviors we intended to express curiosity were also rated as such $(\mathrm{F}[5,228]=53.1879$, $\mathrm{p}<.01)$. Surprisingly, the curious expressions are rated even higher in valence than the expressions designed to communicate positive affect - perhaps our curious clips are overall more energetic and "cute" (tilting head sideways, leaning forward towards the ball) than the positive animations (head bobbing, antenna twirling). However, while the positive and curious motions expressions were both rated as high in valence, the converse was not true - positive motions were not seen as particularly curious.

2) Expressions of intent: Similarly, to evaluate expressions of intent, we asked participants to imagine that they had walked up to Roillo and that the ball was already there. They viewed 3 animations in which the robot uses attentional gestures alone (focusing on the person, then the ball, then the person again), in conjunction with affect (antenna twirling), and in conjunction with deictic gesturing (pointing at the ball with the antenna). An example is illustrated in Fig. 5. For each of the clips, we asked respondents how much they thought Roillo wanted them to pass it the red ball (i.e. the effectiveness of the request). Answers were once again given on a Likert scale.

All three behaviors were rated similarly and differences 


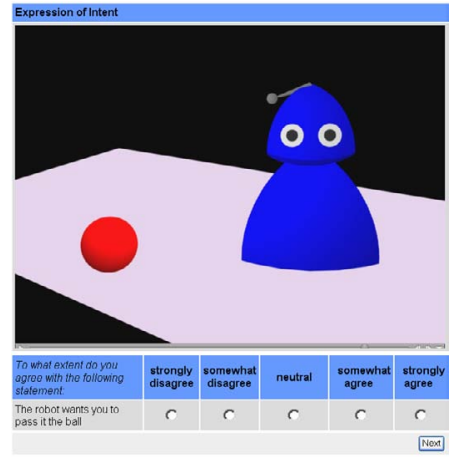

Fig. 5. Roillo requesting the ball using a deictic gesture.

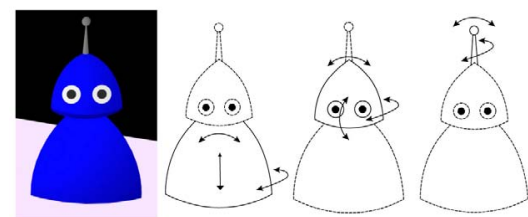

Fig. 6. Desired degrees of freedom for the body, head, and antenna.

were not significant. We had expected deictic motions employing the antenna to be rated as more effective in communicating intent than the affect or attention motions. It is possible that the question and task description were not sufficient for uncovering any differences in perception of these three behaviors, and we intend to revisit this question.

\section{Mechanical design}

Based on these desired behaviors, we identified the degrees of freedom Roillo should have (Fig. 6). The body should rotate and tilt in all directions, the head should rotate, nod, and tilt, and the antenna should swivel through a half-sphere.

However, Roillo is to be simple, inexpensive, and robust to the sort of rough handling that can be expected from children. For these reasons, we avoided designing a complex armature that would be directly driven by servos. Instead, our approach was to use inexpensive servos to pull wires attached to points in a foam body, and to use the springiness of the foam to bring those points back to their starting positions.

To test the feasibility of this approach, we built an approximately $15 \mathrm{~cm}$ tall mechanical prototype from foam, wood, wires, and hobby airplane servos. A wooden segment is attached to the bottom of the head to make it rigid, and wires running through the body are attached to the three lower corners of the head. Three servos mounted below the body are capable of displacing these points by approximately $2 \mathrm{~cm}$. By driving appropriate combinations of the servos, Roillo is able to nod its head up and down, tilt its head left and right, and "bounce" its body up and down (Fig. 7).

The materials and design of the body are quite robust to applied pressure. We believe that this design (pulling on wires running through shape-recovering foam) is useful for designing simple, robust, articulated robots.

\section{Interaction design}

Our next step was to perform an exploratory study aimed at gaining a general sense of children's initial impressions of Roillo - how children perceived and reacted to Roillo's minimal design and to the range of movements the robot would be able to make - before making further investments of time and finances in improving the prototype. Since our prototype enabled only a limited range of the desired motions, we separately constructed a $1 \mathrm{ft}$ tall rubber Roillo puppet (Fig. 8). The puppet sat atop a $2 \times 2 \mathrm{ft}$ box that housed the puppeteer. A one-way mirror allowed the puppeteer to see interactors.

The week-long study was conducted with 22 children, ages 4-6, from a local kindergarten. The children entered the room one by one (13 subjects) or in small groups (9 subjects divided into 4 groups). Upon entering, a researcher instructed the children that they were to "meet Roillo." The researcher inquired about the children's opinions regarding Roillo during and after the interaction. The interactions were videotaped and reviewed by the researchers to find themes in children's behaviors and statements about Roillo.

Along with children's general reactions to Roillo, we were interested in how the rhythm of Roillo's movements and their contingency to the actions of the children affected the interaction. Our aim was to see when and how children engaged in rhythmic entrainment, turn-taking, and imitation. The puppeteer was given free reign in selecting the types of movements and ways to interact with the children, so as to see what kinds of interactive strategies a human would employ using Roillo's expressive capabilities. With this in mind, we created two conditions for the puppeteer - in the first (contingent) case, he was aware of the children's movements by looking through the mirror and responded to them as appropriately as possible; in the second (noncontingent) case, he did not look at the children but moved the puppet around "randomly" while listening to music, which was meant to impart a rhythmic quality to the puppet's actions. Out of 13 interactions with single children, 8 were contingent and 5 were non-contingent. We looked for ways in which contingency affected the rhythmic qualities of the interaction (incidence of children engaging in rhythmic synchrony, turn-taking, or imitation with the puppet) as well as how they affected the children's perception of the puppet.

1) Results: Most subjects (16 out of 22) had a positive impression of Roillo during and at the end of the interaction, while 4 disliked the puppet. Eight of those subjects stated that what they liked most about Roillo were its movements. Many were interested in how it moved, and some suggested
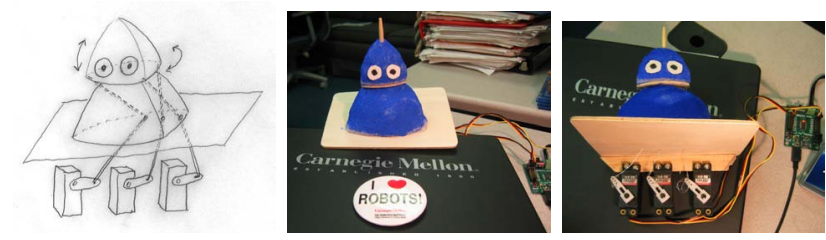

Fig. 7. Our initial mechanical prototype with three servos 


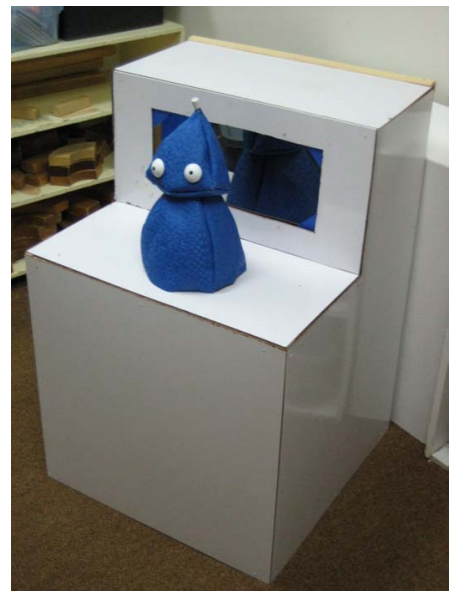

Fig. 8. Our puppet and puppeteer's box.

that "a stick is moving it" (2), that it needed batteries (3) or electricity (1), or its autonomy stayed a mystery (3).

In response to questions about what Roillo is, the most common responses were an alien (5) and a robot (6), although it was also compared to a frog, a teddy bear, a baby, and a bald person. The only female subject that identified Roillo as a robot called it a "baby robot." Two children realized it was a puppet, and 7 thought it was a machine. The antenna was mentioned as a cue to its being a robot or an alien. In the future we plan to experiment with a deictic feature with a less mechanistic appearance.

The children also ascribed meaning and intention to the puppet's actions. A majority of the subjects stated during the interaction that it was looking around the room or at them directly. The subjects also discussed whether Roillo "wants to be touched," is "trying to get out of the hole," or "wants to stay on the box." Some of the subjects interpreted Roillo's movements as representing certain emotions (that it was either happy or scared). Eight subjects thought Roillo was aware of their presence and responding to their actions.

Ten out of the 22 subjects questioned the puppet's minimal design, inquiring as to why it does not have legs, arms, clothing, ears, a nose, or a mouth. Nonetheless, our minimal design successfully conveyed Roillo's sensory and behavioral limitations, because the subjects did not expect Roillo to talk or get up and walk away. The existence of eyes, in both contingent and non-contingent circumstances, did evoke a belief that the puppet could see and was actively looking around the room and focusing on particular people and objects. As expected, the children were very interested in tactile interaction - almost all the subjects touched Roillo from pats, pokes, prods, kisses, and hugs to constant contact.

Regarding rhythmic entrainment, from a preliminary analysis of the video we saw 11 (out of 22) children who engaged with Roillo imitating and synchronizing their movements with the puppet's movements. All the children that engaged rhythmically with the puppet said they liked it, while none of the 5 children who disliked Roillo engaged in rhythmic interaction with the puppet. When they were in groups, the children were more active and developed turn-taking and imitative behavior together.

Our results from comparisons of contingent and noncontingent interactions support the themes discussed in section IIB. Subjects invariably identified the non-contingent movements as "dancing." They noticed that there was something odd about the movement - the puppet was shaking, "moved too much," or "never stops moving." While emotions such as happiness and fear were attributed (5 times) to Roillo when it moved contingently, the non-contingent motion did not evoke any emotive comparisons. Only one of the subjects involved in non-contingent interaction perceived Roillo as being aware of and responsive to his presence, while 7 children in contingent interactions expressed awareness of its responsiveness. Similarly, of the 8 subjects who liked Roillo's movements, only one was involved in noncontingent interactions - and he was the only one that engaged in rhythmic synchrony with the puppet while it was behaving in a non-contingent manner.

In summation, our exploratory study of childrens interactions with Roillo provided positive evaluations of Roillo's physical and interactive design and gave results consistent with previous research on interaction rhythms. The minimal design was successful in representing the puppet's sensory and behavioral abilities. Children's evaluations were in line with previous results correlating contingency and rhythmic synchrony with subjects' positive evaluations of the interaction partner. Non-contingent interactions were generally negatively evaluated, and did not evoke impressions of Roillo as an intentional, emotional, or conscious agent.

\section{Current \& Future Work}

\section{A. Physical design}

Our next step in the physical design of the system will be to create a new "skin" for the robot, keeping to the size of our puppet. As we move forward in the design process and refine the actuation of the robot, it will be useful to be able to puppeteer the skin for the purposes of demonstration or further testing. We intend to 3D print a model of Roillo, create a mold, and cast the skin from a rubber such as latex.

The skin will be filled with a foam for structure and protection from rough handling. We will actuate the robot either by our method of pulling on wires (and adding an appropriate rigid skeleton for directing the forces in the desired directions), or by embedding servos directly in the body, or by a combination of the two methods.

\section{B. Perception of rhythm}

Our exploratory study of Roillo with children shows that contingency and rhythmicity of interaction have a significant effect on perception of and interaction with a social robot or puppet. However, the difficulty of achieving autonomous rhythmic synchrony stems largely from the difficulty in perceiving what are often small changes in visual or auditory stimuli. Research in speech patterns is rather extensive, but motion is relatively unexplored.

We intend to approach the problem of rhythmic motion perception from two angles. First, we will look at how successfully we can detect oscillatory or repetitive movements in 
the environment from a camera using simple vision processes (e.g. face detection, skin detection, feature tracking. etc.). Crick [8] detected extremities in the spatial movement of the skin color of a "conductor's" hand, and we can imagine doing the same with head or body movements. Second, in order to obtain more accurate information about human body movement during social interaction, we can look for repetitive or rhythmic movements in motion capture data. Ogawa [21] has developed a robotic telepresence system that allows two remote humans to communicate with each other through a pair of robots. The speech is transmitted directly, and the robots' physical behaviors are controlled by models of speech/motion correlation obtained from human speakers and listeners. They measure head, arm, and body motions using magnetic sensors, which provide similar data to motion capture data and are a promising alternative method.

\section{Dance as an application}

A typical human activity in which the perception of rhythmicity is made explicit is dance, and specifically dance instruction. We intend to observe dancers and instructors, how they synchronize, how an instructor corrects a student's errors, etc. We can envision a task in which the robot tries to teach a child a particular dance and can evaluate whether the child is doing it correctly. We can also explore dance as an interactive social activity that the robot can use to study a person's behavioral patterns and identify rhythmic abnormalities that could be useful in diagnosing certain pathologies. Dance has, in turn, been identified as a potentially therapeutic activity [15].

\section{CONCLUSiON}

We have introduced Roillo, a social robotic platform for the investigation of nonverbal behaviors in the context of interaction with children. We believe that being able to perceive, generate, and synchronize with rhythmic behavior is an important and underestimated basis for the sophisticated behaviors we would like social robots to exhibit.

We have used a design process that involves a number of tools and methodologies. Through surveys about graphical renderings and animations, we created a minimally designed character and explored a number of behaviors that convey affect, attention, and intent. Given the application requirements of building a toy for children, we have tested a mechanical design that involves inexpensive motors pulling on foam. Finally, we created a puppet of the robot and observed, in interactions with children, a range of interesting behaviors and found clear effects of contingency and rhythmic movement on children's behavior and attributions. We intend to continue this work with the aim of creating an autonomous Roillo that can perceive and appropriately respond to the rhythmic behaviors of interaction partners and can be used in more rigorous experiments on the effects of rhythmicity in human social interaction.

\section{ACKNOWLEDGMENTS}

This project began in the CMU course 16899, Principles of Human-Robot Interaction. This project was partially sup- ported by National Science Foundation ITR projects \#IIS0329014, \#SES-0522630, and a GRFP. The authors would like to thank Linnda R. Caporael, Illah Nourbakhsh, Brian Scassellati, and Reid Simmons for helpful comments.

\section{REFERENCES}

[1] P. Andry, P. Gaussier, S. Moga, J. P. Banquet, and J. Nadel. Learning and communication via imitation: An autonomous robot perspective. IEEE Transactions on Systems, Man, and Cybernetics, 31(5), September 2001 .

[2] A. G. Brooks and C. Breazeal. Working with robots and objects: Revisiting deictic reference for achieving spatial common ground. In Proceedings of Human-Robot Interaction, pages 297-304, 2006.

[3] E. Chapple. Movement and sound: The musical language of body rhythms in interaction. In M. Davis, editor, Interaction Rhythms: Periodicity in Communicative Behavior. Human Sciences Press, New York, 1982.

[4] T. Chartrand and J. Bargh. The chameleon effect: The perceptionbehavior link and social interaction. Journal of Personality and Social Psychology, 76:893-910, 1999.

[5] W. Condon. Communication: Rhythm and structure. In J. Evans and M. Clynes, editors, Rhythms in Psychological, Linguistic and Musical Processes. Charles C. Thomas Publisher, Springfield, 1986.

[6] W. Condon and W. Ogston. Sound film analysis of normal and pathological behavior patterns. Journal of Nervous and Mental pathological behavior pattern
Disease, 143:338-347, 1966.

[7] W. Condon and L. Sander. Neonate movement is synchronized with adult speech: Interactional participation and language acquisition. Science, 183(4120):99-101, 1974.

[8] C. Crick, M. Munz, T. Nad, and B. Scassellati. Robotic drumming: synchronization in social tasks. Under review, 2006.

[9] K. Dautenhahn. Robots as social actors: Aurora and the case of autism. In Proc. if the International Cognitive Technology Conference, 1999.

[10] T. Fong, I. Nourbakhsh, and K. Dautenhahn. A survey of socially interactive robots: Concepts, design and applications. Technical Report CMU-RI-TR-02-29, Carnegie M
Pittsburgh, PA, November 2002.

[11] E. Goffman. Interaction Ritual: Essays on Face-to-Face Behavior. Anchor Books, New York, 1967.

[12] E. Hatfield, J. Cacioppo, and R. Rapson. Emotional Contagion. Cambridge University Press, New York, 1994

[13] S. Johnson, V. Slaughter, and S. Carey. Whose gaze will infants follow? the elicitation of gaze-following in 12-month-olds. Journal of Developmental Science, 1(2):233-238, 1998.

[14] M. Jones. Time, our lost dimension: Toward a new theory of perception, attention and memory. Psych. Review, 83:323-353, 1976.

[15] B. Kalish. Body movement therapy for autistic children. Proceedings of the 3rd annual conference of the American Dance Therapy Association, 1968.

[16] A. Kendon. Movement coordination in social interaction: Some examples described. Acta Psychologica, 32:100-125, 1970.

[17] H. Kozima. Infanoid: A babybot that explores the social environment. In K. Dautenhahn, A. H. Bond, L. Canamero, and B. Edmonds, editors, Socially Intelligent Agents: Creating Relationships with Computers and Robots, pages 157-164. Kluwer Academic Publishers, 2002.

[18] H. Kozima, C. Nakagawa, Y. Yasuda, and D. Kosugi. A toy-like robot in the playroom for children with developmental disorders. In Proceedings of the International Conference on Development and Proceedings of the International
Learning, San Diego, CA, 2004.

[19] M. LaFrance. Posture mirroring and rapport. In M. Davis, editor, Interaction Rhythms: Periodicity in communication behavior. Human Sciences Press, New York, 1982.

[20] F. Michaud and C. Theberge-Turmel. Mobile robotic toys and autism. In K. Dautenhahn, A. Bond, L. Canamero, and B. Edmonds, editors, Socially Intelligent Agents - Creating Relationships with Computers and Robots, pages 125-132. Kluwer Academic Publishers, 2002.

[21] H. Ogawa and T. Watanabe. Interrobot: speech-driven embodied interaction robot. Advanced Robotics, 15:371-377, 2001.

[22] M. Okada, S. Sakamoto, and N. Suzuki. Muu: Artificial creatures as an embodied interface. In ACM SIGGRAPH Conference Abstracts and Applications, page 91, New Orleans, 2000.

[23] S. Penny. Embodied cultural agents: at the intersection of robotics, cognitive science, and interactive art. In AAAI Socially Intelligent Agents Symposium, 1997.

[24] C. Plaisant, A. Druin, C. Lathan, K. Dakhane, K. Edwards, and J. Vice. A storytelling robot for pediatric rehabilitation. In Proceedings of ASSETS 2000, Washington DC, November 2000.

[25] S. Restivo. Romancing the robots: Social robots and society. In Robot as Partner: An Exploration of Social Robots Workshop, International as Partner: An Exploration of Social Robots Workshop,

[26] C. Trevarthen. Communication and cooperation in early infancy: A description of primary intersubjectivity. In M. Bulowa, editor, Before speech: The beginning of human communication, pages 321347. Cambridge University Press, London, 1979.

[27] C. Trevarthen. Can a robot hear music? can a robot dance? can a robot tell what it knows or intends to do? can it feel pride or shame in company? - questions of the nature of human vitality In Proceedings of the Second International Workshop on Epigenetic Robotics: Modeling Cognitive Development in Robotic Systems, 2002

[28] E. Tronick and J. Cohn. Infant-mother face-to-face interaction: Age and gender difference in coordination and the occurrence of miscoordination. Child Development, 60(1):85-92, 1989. 